\title{
A novel splice-site mutation in RHD gene associated with RhD negative phenotype
}

\author{
Maria Antonietta Villa, Nicoletta Revelli, Cinzia Paccapelo, \\ Francesca Truglio, Araitz Molano, Mónica López
}

\begin{abstract}
Introduction: The $\mathrm{Rh}$ antigens are encoded by the highly polymorphic RHD and the RHCE genes. Individuals with $D$ variants may make anti-D alloantibodies, which in pregnant women cause severe or fatal hemolytic disease of the fetus and newborn. Accurate $D$ typing using reliable routine methods must be performed. A number of unusual RHD alleles are being currently reported due in large part to the growing implementation of molecular analyses. Case Report: A sample from a Caucasian male blood donor at Northern Italy was serologically classified as RhD negative (Ccee). Instead, the sample was genotyped with all SSP-PCR kits as RhD positive, Dd and the result obtained with Partial D-TYPE (Dva or Va like or Va associated or DBS) was consistent with a suggestion of a new variant. Through a microarray platform analysis (BLOODchip Reference), the sample was genotyped as DV/DBS [RHD-CE(S)-D hybrid], associated with a predicted partial $D$ phenotype for the RhD group, due to the lack of
\end{abstract}

Maria Antonietta Villa ${ }^{1}$, Nicoletta Revelli ${ }^{1}$, Cinzia Paccapelo ${ }^{1}$ Francesca Truglio', Araitz Molano², Mónica López ${ }^{2}$

Affiliations: 'Laboratorio di Immunoematologia di Riferimento - U.O.C. Centro Trasfusionale, Fondazione IRCCS Ca' Granda Ospedale Maggiore Policlinico, Milan, Italy; ${ }^{2}$ Progenika Biopharma, a Grifols Company, Derio, Spain.

Corresponding Author: Maria Antonietta Villa, ScD, Laboratorio di immunoematologia di Riferimento U.O.C. Centro Trasfusionale Fondazione IRCCS Ca' Granda Ospedale Maggiore Policlinico Via F. Sforza, 35, 20122 Milano, Italy; Email: a.villa@policlinico.mi.it

Received: 24 October 2017

Accepted: 20 November 2017

Published: 28 December 2017 a hybridization signal for RHD exon 5 . The PCR analysis of the exon 5 of the RHD gene gave a positive amplification result and the sequencing revealed a polymorphism in hemizygous or homozygous state in position RHD*IVS5+1t. This novel splice-site mutation alters or completely abolishes the specific sequence where the splicing of RHD gene intron 5 takes place. Conclusion: The novel RHD mutation hereby described highlights the significance of using RHD genotyping to confirm and/or clarify $D$ antigen uncertainties in order to prevent the unnecessary immunization of patients.

Keywords: D variants, Novel RHD mutation, Sequencing

\section{How to cite this article}

Villa MA, Revelli N, Paccapelo C, Truglio F, Molano A, López M. A novel splice-site mutation in RHD gene associated with RhD negative phenotype. Int J Blood Transfus Immunohematol 2017;6:46-50.

Article ID: 100034IJBTIMV2017

$$
* * * * * * * * *
$$

doi: 10.5348/ijbti-2017-34-CR-7

\section{INTRODUCTION}

The $\mathrm{Rh}$ blood group is one of the most complex and polymorphic systems of red blood cells (RBCs) known in humans. Due to its high immunogenicity the Rh antigens play a crucial role in transfusion medicine, particularly in obstetrics, as they are the main cause of hemolytic 
disease of the fetus and newborn (HDFN) [1]. The Rh antigens expressed on the red blood cell (RBC) surface are defined by a trimeric complex of membrane polypeptides encompassing the $\mathrm{Rh}$ proteins and the $\mathrm{Rh}$-associated glycoprotein (RhAG). The Rh antigens are encoded by two highly polymorphic genes located on the short arm of chromosome 1, the RHD and the RHCE genes. Each of these genes, originating from a duplication of a common gene ancestor, are comprised of 417 amino acids and 10 exons, form the RH locus. The RHD gene encodes the RhD protein, whereas the RHCE gene encodes the RhCcEe polypeptides carrying the $\mathrm{C}(\mathrm{RH} 2) / \mathrm{c}\left(\mathrm{RH}_{4}\right)$ and $\mathrm{E}\left(\mathrm{RH}_{3}\right) / \mathrm{e}\left(\mathrm{RH}_{5}\right)$ antigens [2].

Due to their similarity, close proximity and opposite orientation, the two genes promote numerous genetic rearrangements, giving rise to variant alleles. The D variants encoded by these alleles can arise from different mechanisms such as nucleotide insertions or deletions, single or multiple missense mutations, splice site mutations, and gene conversions [3, 4]. The RHD alterations, affecting a partially or exclusively extracellular $\mathrm{RhD}$ section, result in the partial $\mathrm{D}$ variants, leading to type-specific D epitope lacking or diminishing the D antigen density. The partial $\mathrm{D}$ variants include partial $\mathrm{D}$, weak D and DEL types, and their identification is of crucial importance in transfusion medicine [5]. Individuals with partial D variants may make alloantibodies anti-D against those D epitopes, sometimes in pregnant women causing severe or fatal HDFN [6-8]. Most RBCs known as weak D are thought to express D antigens altered in a quantitatively, but not qualitatively, form. Therefore, such individuals appear not to develop alloantibodies anti-D upon contact with normal D positive RBCs.

Reduced expression of $\mathrm{D}$ antigen, quantitatively or qualitatively, occurs in $0.3^{-1 \%}$ of Caucasian people. Although the most common cause of the $\mathrm{D}$ negative in people of European origin is homozygosity for a complete deletion of RHD gene, the association of more than 115 mutations and RHD-RHCE gene conversion with this phenotype has also been described [9].

In past years, a number of unusual RHD alleles have been reported due to the growing use of several different known D epitope specificity monoclonal antibodies [10] and the evaluation of RHD alleles by the implementation of molecular analyses [11]. For that reason, D typing using reliable routine $\mathrm{D}$ typing methods must be performed in mother-to-be transfusion recipients, with special care in the case of blood donors with weak expression variants.

\section{CASE REPORT}

We describe a case of weak reactivity of $\mathrm{D}$ antigen found in a Caucasian male blood donor resulting from nucleotide change. The sample was sent, after obtaining a signed informed consent, to our Immunohematology Reference Laboratory (IRL) due to incongruent results detected in RhD typing from the Northern Italy Transfusion Blood
Centre. Routine serological D typing was performed in hemagglutination with a fully automated system (Galileo, Immucor, Norcross, GA, USA) at room temperature by immediate spin employing a liquid-phase in microplate method (Select R, Immucor) using two different blended monoclonal reagents, the DUO IgM TH28 + IgG MS26 and NOVACLONE D175+D415 clones (Immucor). The sample was further tested by Innova Instrument (Ortho Biovue System, Ortho Clinical Diagnostics Raritan, NJ, USA) employing the ABOD1D2 card (Ortho Clinical Diagnostics) in column agglutination technology (CAT) using two anti-D monoclonal antibodies D7B8 and RUM1 (Ortho). The Weak D indirect antiglobulin test (IAT) was performed in solid-phase red cells adherence (SPRCA) with the same reagents of the microplate method and in CAT with the anti-D clones D7B8+H1121G6 +LORIFA (Ortho Clinical Diagnostics).

Extended $\mathrm{Rh}(\mathrm{D})$ typing for epitope detection was done using Galileo (Immucor) with the ALBAcloneR Advanced Partial RhD typing kit (Albabioscience, Ellen's Glen Road, Edinburgh, UK) to differentiate partial RhD types and weak RhD types 1 and 2. The phenotype was determined using the microplate method (Galileo) with monoclonal antibodies specific for Rh antigens (CcEe). All tests were performed according to the manufacturers' recommendations. These devices and reagents have $\mathrm{CE}$ Marking license in Europe.

A bench top apparatus for fully automated nucleic acid purification (QIACube-Qiagen, Hilden, Germany) was used, according to the manufacturer's instructions, to extract DNA from whole blood cells. DNA analysis was performed using single specific primer-polymerase chain reaction (SSP-PCR) with Ready Gene CDE kit (Inno-Train Diagnostik, GmbH, Kronberg im Taumus, Germany) and RH-TYPE, Weak D-TYPE, Partial D-TYPE kits (BAGene, BAG Health Care GmBH, Lich, Germany). Subsequently, to perform extended typing, the sample was sent to the Grifols-Progenika Reference Laboratory and tested by a microarray platform (BLOODchip Reference v4.o, Progenika Biopharma, Derio, Spain) designed to detect 128 polymorphisms, including RHD and RHCE variants. The result of BLOODchip Reference v4.o test was further confirmed with DNA by the Sanger method [12].

Serologically, the sample was classified as RhD negative (Ccee). The reaction pattern of monoclonal anti-D's is reported in Table 1 . Instead, the sample was genotyped with all SSP-PCR kits as RhD positive, Dd and the result obtained with Partial D-TYPE (Dva or Va like or Va associated or DBS) was consistent with a suggestion of a new variant.

Through the BLOODchip Reference v4.o analysis, the sample was genotyped as DV/DBS [RHD-CE(S)-D hybrid], associated with a predicted partial D phenotype for the RhD group, due to the lack of a hybridization signal for RHD exon 5. Thereafter, the sequencing of RHD exon 5 with an alternative primer pair was performed, to check its presence/absence and its sequence. In addition, the sequencing of RHD gene exons 1-4 and 6-10 was also 
carried out to search for any additional mutation not included in the BLOODchip Reference v4.o, and which could explain the serology results. The PCR analysis of the exon 5 of the RHD gene gave a positive amplification result and the sequencing revealed a polymorphism in hemizygous or homozygous state in position RHD*IVS5+1t. This nucleotide change is located in the BLOODchip Reference v4.o RHD exon 5 primer reverse sequence, and it explains the lack of amplification; the rest of exon 5 sequence was consensus RHD. Sequencing of exons 1-4 and 6-10 of RHD gene did not reveal additional mutations (Figure 1 ).

\section{DISCUSSION}

Considering weak $\mathrm{D}$ and partial $\mathrm{D}$ with frequencies lower than $1 \%$, even if in the daily practice of most blood transfusion centers the status of $\mathrm{Rh}(\mathrm{D})$ antigens are determined by serology with agglutination tests. For the immunogenicity of $\mathrm{D}$ antigen and the high rate of immunization of D-negative individuals after the transfusion of D-positive RBCs, the determination of

Table 1: Reaction pattern of monoclonal anti-D clones

\begin{tabular}{llll} 
Monoclonal anti-D & Patient RBCs \\
\hline Reagent & Clones & IS & IAT \\
Ortho D1 & D7B8 & o & Not tested \\
Ortho D2 & RUM1 & o & Not tested \\
Ortho D3 & D7B8+H1121G6+LORIFA & o & o \\
Immucor DUO & TH28+MS26 & o & Weak \\
Immucor & D175+D415 & o & Weak \\
NOVACLONE & & & \\
ALBAclone $\mathbb{R}$ & LHM76/58; LHM76/59; & o & o \\
Advanced & LHM174/102; & & \\
Partial RhD & LHM50/2B; LHM169/81; & & \\
& ESD1; LHM76/55; & & \\
& LHM77/64; LHM70/45; & & \\
& LHM59/19; LHM169/80; & & \\
& LHM57/17 & & \\
\hline
\end{tabular}

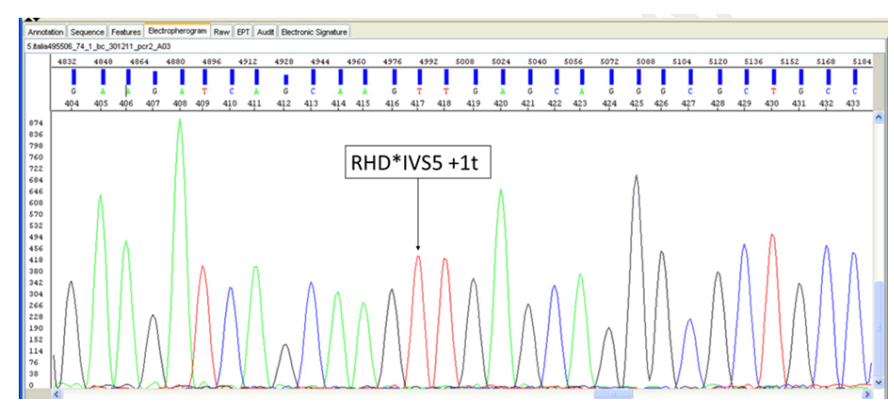

Figure 1: Sequencing analysis of RHD exon 5 and its adjacent intronic regions. Highlighted the nucleotide hemizygous mutation found at position $\mathrm{IVS}_{5}+1, \mathrm{RHD}^{*} \mathrm{IVS}_{5}+1 \mathrm{t}$, located in BLOODchip reference v4.0 RHD exon 5 primer reverse sequence.
RHD alleles play a significant role in D negative pregnant women carrying D positive fetuses and in blood donors. Pregnant women expressing weak D type alleles or carrying $\mathrm{D}$ negative fetuses, which can be detected by RHD genotyping, can avoid RhIg prophylaxis [13]. Instead, RHD genotyping in blood donors is beneficial to transfusion recipients because it can exclude weak D, partial D and DEL donors among apparent D negative blood donors and avoid red cell alloimmunization. It is obvious that determining a donor's phenotype and genotype is more decisive to obtain correct results than two or more serological tests alone [14].

Herein, we report the molecular background of a novel D mutation and their associated immunohematological features. This novel splice-site mutation alters or completely abolishes the specific sequence where the splicing of RHD gene intron 5 takes place. Therefore, the protein product would be aberrant explaining the RhD negative serology result. This hypothesis is supported by the previous description of another nucleotide change in the same position (RHD*IVS $5+1 \mathrm{~g}>\mathrm{a}$ ) which is also associated with a D negative phenotype in the Portuguese population $[15,16]$.

\section{CONCLUSION}

The novel RHD mutation, RHD*IVS $5+1$ t hereby described should affect the splicing site and generate an $\mathrm{RhD}$ negative phenotype and explain the serology results. In fact, at first, serological analysis for D appeared to be trustworthy negative and only the use of the genetic protocol was decisive for obtaining correct results. Strategies in blood banks regarding the weak D types and the immunization should be established, to reliably type these individuals as RhD-positive, not prone to anti-D immunization. The application of methods based on molecular biology could lead to a rapid and cost-effective large-scale detection in the near future. This case report highlights the significance of using RHD genotyping to confirm and/or clarify D antigen uncertainties in order to prevent the unnecessary immunization of patients.

\section{REFERENCES}

1. Mollison PL, Klein HG, Anstee DJ. Mollison's Blood Transfusion in Clinical Medicine. 12ed. Oxford, UK: Wiley-Blackwell; 2014.

2. Daniels G. Biochemistry and molecular genetics of the Rh polypeptides. In: Daniels G, editor. Human Blood Groups. 3ed. Oxford, UK: Wiley-Blackwell; 2013.

3. Avent ND, Reid ME. The Rh blood group system: A review. Blood 2000 Jan 15;95(2):375-87.

4. Flegel WA. Blood group genotyping in Germany. Transfusion 2007 Jul;47(1 Suppl):47S-53S.

5. Daniels G. Variants of RhD: Current testing and clinical consequences. $\mathrm{Br} \mathrm{J}$ Haematol 2013 May;161(4):461-70. 
6. Cannon M, Pierce R, Taber EB, Schucker J. Fatal hydrops fetalis caused by anti-D in a mother with partial D. Obstet Gynecol 2003 Nov;102(5 Pt 2):11435 .

7. Lacey PA, Caskey CR, Werner DJ, Moulds JJ. Fatal hemolytic disease of a newborn due to anti-D in an Rh-positive $\mathrm{Du}$ variant mother. Transfusion 1983 Mar-Apr;23(2):91-4.

8. Ostgård P, Fevang F, Kornstad L. Anti-D in a 'D positive' mother giving rise to severe haemolytic disease of the newborn. A dilemma in antenatal immunohaematological testing. Acta Paediatr Scand 1986 Jan;75(1):175-8.

9. Wagner FF, Flegel WA. The rhesus site. Transfus Med Hemother 2014 Oct;41(5):357-63.

10. Wagner FF, Frohmajer A, Ladewig B, et al. Weak D alleles express distinct phenotypes. Blood $2000 \mathrm{Apr}$ 15;95(8):2699-708.

11. Flegel WA, Wagner FF. Molecular biology of partial $\mathrm{D}$ and weak D: Implications for blood bank practice. Clin Lab 2002;48(1-2):53-9.

12. Sanger F, Coulson AR. A rapid method for determining sequences in DNA by primed synthesis with DNA polymerase. J Mol Biol 1975 May 25;94(3):441-8.

13. Flegel WA. How I manage donors and patients with a weak D phenotype. Curr Opin Hematol 2006 Nov;13(6):476-83.

14. Flegel WA. Molecular genetics and clinical applications for RH. Transfus Apher Sci 2011 Feb;44(1):81-91.

15. https://www.ncbi.nlm.nih.gov/nuccore/JQ846090

16. Gassner C, Doescher A, Drnovsek TD, et al. Presence of RHD in serologically D-, C/E+ individuals: A European multicenter study. Transfusion 2005 Apr;45(4):527-38.

\section{$* * * * * * * * *$}

\section{Acknowledgements}

Serena Scognamiglio and Maurizio Marconi (Laboratorio di Immunoematologia di Riferimento) and Paola Ponzo (Grifols) are acknowledged for their critical review of the manuscript. Jordi Bozzo PhD CMPP (Grifols) is acknowledged for critical review and editorial support in the preparation of the manuscript.

\section{Author Contributions}

Maria Antonietta Villa - Substantial contributions to conception and design, Acquisition of data, Analysis and interpretation of data, Drafting the article, Revising it critically for important intellectual content, Final approval of the version to be published

Nicoletta Revelli - Substantial contributions to conception and design, Acquisition of data, Analysis and interpretation of data, Drafting the article, Revising it critically for important intellectual content, Final approval of the version to be published

Cinzia Paccapelo - Substantial contributions to conception and design, Acquisition of data, Analysis and interpretation of data, Drafting the article, Revising it critically for important intellectual content, Final approval of the version to be published

Francesca Truglio - Substantial contributions to conception and design, Acquisition of data, Analysis and interpretation of data, Drafting the article, Revising it critically for important intellectual content, Final approval of the version to be published

Araitz Molano - Substantial contributions to conception and design, Acquisition of data, Analysis and interpretation of data, Drafting the article, Revising it critically for important intellectual content, Final approval of the version to be published

Mónica López - Substantial contributions to conception and design, Acquisition of data, Analysis and interpretation of data, Drafting the article, Revising it critically for important intellectual content, Final approval of the version to be published

\section{Guarantor of Submission}

The corresponding author is the guarantor of submission.

\section{Source of Support}

None

\section{Conflict of Interest}

Authors declare no conflict of interest.

\section{Copyright}

(C) 2017 Maria Antonietta Villa et al. This article is distributed under the terms of Creative Commons Attribution License which permits unrestricted use, distribution and reproduction in any medium provided the original author(s) and original publisher are properly credited. Please see the copyright policy on the journal website for more information. 
Access full text article on other devices

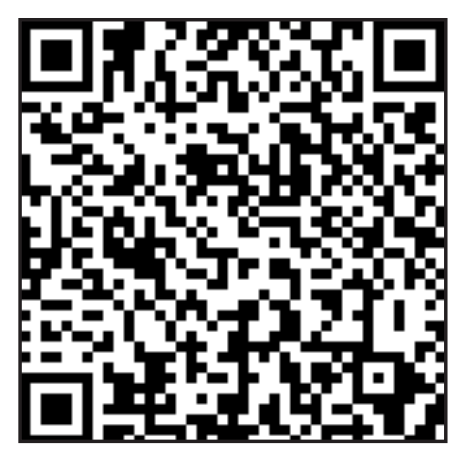

Access PDF of article on other devices

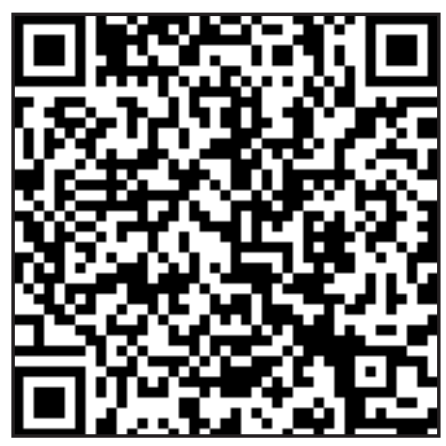

